\title{
Evaluation of the Papanicolaou-stained cytological smear as a screening technique for asymptomatic gonorrhoea
}

\author{
GAIL I GENVERT,* LEWIS M DRUSIN,*†‡ JOHN F SEYBOLT, §॥ AND \\ DAVID W BRAUN
}

From the Departments of + Public Health, $¥$ Medicine, and §Pathology, Cornell University Medical College; the *Department of Epidemiology and the IIPapanicolaou Cytology Laboratory, New York Hospital; and the \Biostatistics Laboratory, Memorial Sloan-Kettering Cancer Center, New York, USA

SUMMARY To assess the Papanicolaou smear as a screening test for asymptomatic gonorrhoea 311 women who had had simultaneous Papanicolaou smears and cervical cultures performed were studied. Of the 151 women who were culture-positive only $27(18 \%)$ had a positive result by Papanicolaou smear. The presence of either endocervical or metaplastic cells did not increase the frequency with which gonococci were recognised. The eight cytotechnologists spent at least 30 minutes for each case looking for diplococci; the variability between observers was high.

The possibility of using an already widely accepted diagnostic technique, such as the cytological smear, as a means of screening for gonococci is very attractive. Unfortunately in this study the excessive time taken to examine the slides, the poor correlation of observers' findings, and a low sensitivity for finding diplococci made the Papanicolaou smear an impractical method for detecting gonorrhoea in asymptomatic women.

\section{Introduction}

Gonorrhoea is the most frequently reported communicable disease in the United States. During 1977, the Center for Disease Control reported 1000177 cases and estimated the actual incidence of gonorrhoea at 1·6-2.0 million cases annually. ${ }^{1}$ In women, culture of samples from both the cervix and rectum on Thayer-Martin media is the recommended method for making the diagnosis of gonorrhoea. ${ }^{2}$ Performing bacterial cultures on a large population of women at risk for this disease is both expensive and inconvenient. Public health authorities have been searching for a more practical and socially acceptable method of identifying asymptomatic cases. Recently, Neisseria gonorrhoeae has been recognised on Papanicolaou smears performed on women with active gonococcal infections. ${ }^{34}$ These investigators have suggested that the Papanicolaou smear might be useful in detecting asymptomatic gonococcal carriers among those women who undergo an annual gynaecological examination.

Address for reprints: Dr L M Drusin, Department of Public Health, The New York Hospital-Cornell Medical Center, 525 East 68th Street, New York, NY 10021, USA

Received for publication 12 March 1980
In this study, the parameters that define a successful screening test (sensitivity, specificity, validity, yield, reliability, and practicality) were applied to data from women who had simultaneous cytological smears and cervical cultures performed at the New York Hospital to evaluate the Papanicolaou smear as a screening test for asymptomatic gonorrhoea.

\section{Patients and methods}

\section{CULTURES}

Cervical cultures for gonorrhoea were performed on Thayer-Martin medium according to the methods recommended by the Center for Disease Control. ${ }^{5}$ Cervical smears were fixed in Spray-cyte (ClayAdams) and stained according to the Papanicolaou technique. ${ }^{6}$

\section{STUDY POPULATION}

The study population consisted of women seen in the department of obstetrics and gynaecology at the New York Hospital who had both a Papanicolaou smear of the cervix and a cervical culture for gonorrhoea performed during a routine examination. A patient was included as a study case if her cervical culture had given a positive result for $N$ gonorrhoeae; 
however, she was included as a control if her cervical culture result was negative. Cases were identified from the gonococcal reporting forms submitted by the diagnostic microbiology laboratory at the New York Hospital to the New York City Department of Health. Controls were located from a computer printout listing all elective abortions performed at the New York Hospital. Charts on these patients were reviewed retrospectively to identify a study population consisting of an approximately equal number of cases and controls.

Names and history numbers of all these patients were arranged consecutively according to the Papanicolaou laboratory's slide accession number, which afforded a random order for the results of the gonococcal cultures.

\section{SCREENING}

Papanicolaou smears from the entire study population were reviewed in a double-blind fashion by a team of eight observers. These were licensed cytotechnologists with an average of 8.8 years of experience (range 4-15 years) of examining cytological smears at the Papanicolaou cytology laboratory. The observers were each shown good examples of smears with gonococci before the study began. They initially scanned the slides at about $\times 150$ magnification $(\times 10$ objective with $\times 15$ eyepieces). If they noted cells with a collection of bacteria they switched to the high dry objective $(\times 40)$ for confirmation. This area was marked with a dot. Finally, to verify the presence of diplococci further, they examined the marked areas under oil at a magnification of $\times 1440$. They were also asked to note the presence of metaplastic and endocervical cells in addition to diplococci. The observers were limited to 30 minutes for each case, for which there were three slides (vaginal, cervical, and endocervical). The reliability of this examination was subsequently evaluated by each of the eight observers independently reviewing the Papanicolaou smears from the same set of randomly selected 20 patients (10 cases and 10 controls).

\section{Results}

A total of 311 women who had had simultaneous Papanicolaou smears and cervical cultures performed over a 40-month period (January 1974 to May 1977) were studied. There were 151 cases and 160 controls. Cases and controls were not compared for demographic characteristics, since the results of the Papanicolaou smears and gonococcal cultures were not dependent on these factors.

The time spent screening slides for gonococci was compared with the time that cytotechnologists
TABLE I Time spent screening routine Papanicolaou smears compared with those for gonorrhoea

\begin{tabular}{|c|c|c|c|}
\hline \multirow[b]{2}{*}{ Interpretation } & \multicolumn{2}{|c|}{ Routine cytology } & \multirow{2}{*}{$\frac{\text { Gonorrhoed }}{\text { Time taken }}$} \\
\hline & $\begin{array}{l}\% \text { of } \\
\text { results† }\end{array}$ & $\begin{array}{l}\text { Time taken } \\
\text { (mins) }\end{array}$ & \\
\hline \multirow{3}{*}{$\begin{array}{l}\text { Clearly negative } \\
\text { Clearly abnormal } \\
\text { Difficult to } \\
\text { interpret }\end{array}$} & 99.00 & $7 \cdot 5$ & \multirow{3}{*}{$\begin{array}{l}30 \text { (for each } \\
\text { case) }\end{array}$} \\
\hline & 0.90 & 25 & \\
\hline & 0.09 & 50 & \\
\hline
\end{tabular}

*Calculated from the mean number of slides with negative results screened per hour by an experienced cytotechnologist

tEstimated from the total number of cases examined in one month

usually take to screen for cytological abnormalities (table I). Clearly, looking for diplococci on a Papanicolaou smear takes an inordinate amount of time. Although the observers invariably used their allotted $\mathbf{3 0}$ minutes for each case in their search for gonococci, they often found that they could have examined the slides for a longer period of time.

\section{SENSITIVITY AND SPECIFICITY}

The overall results are summarised in table II. Of the 151 women who were culture-positive for $N$ gonorrhoeae only $27(\mathbf{1 8 \%})$ had positive results on the Papanicolaou smear. Thus, the sensitivity was very low. Of the 160 women who were culture-negative for $N$ gonorrhoeae all but four had results correctly identified as negative on the cytological smear-a specificity of $97 \%$.

\section{STATISTICAL ANALYSIS}

Given the results of a standard $\chi^{2}$ test, we reject the null hypothesis of no correlation between the Papanicolaou smear and the cervical culture in the detection of gonorrhoea. However, this agreement is not clinically significant. It only reflects the fact that observers regarded $90 \%(280 / 311)$ of all results of Papanicolaou smears reviewed as negative, giving the false impression that there is good agreement between the cytological smear and cervical culture in detecting women who do not have gonorrhoea. This, in turn, is reflected in the high specificity (97\%) noted above, and it is only for this reason that our $P$ value is significant $(P<0 \cdot 001)$. The Papanicolaou

TABLE II Presence or absence of gonococci detected by Papanicolaou smears and cervical cultures

\begin{tabular}{llcc}
\hline & \multicolumn{3}{l}{ Cervical culture results } \\
\cline { 2 - 4 } Smear results & + & - & Total \\
\hline+ & 27 & 4 & 31 \\
Total & 124 & 156 & 280 \\
\hline
\end{tabular}

+ Positive - negative 
smear as a screening test is simply not sensitive enough to pick up cases or carriers with any regularity despite a seemingly good statistical agreement between the two methods of detection.

\section{METAPLASTIC AND ENDOCERVICAL CELLS}

The sensitivity rate for each combination of presence or absence of metaplastic or endocervical cells for those smears taken from culture-positive patients is given in table III. A statistically significant difference was observed when metaplastic cells were presentthat is, a sensitivity rate of $24 \%$ compared with $0 \%$ $(\mathrm{P}<0.01$; Fisher's exact test). This difference is not important however because this low sensitivity is clinically unacceptable. Although there appeared to be some increased sensitivity when endocervical cells were present (27\% compared with $14 \%)$, this difference was not statistically significant $(P>0 \cdot 1)$. When only slides with positive results for metaplastic cells are considered, the small difference associated with the presence of endocervical cells $(29 \%$ compared with $20 \%$ ) was also not statistically significant $(\mathrm{P}>\mathbf{0} \cdot \mathbf{1})$.

TABLE III Percentage of Papanicolaou smears showing gonococci in relation to presence or absence of endocervical and metaplastic cells from patients with positive cervical culture results

\begin{tabular}{lccc}
\hline & \multicolumn{2}{l}{$\%$ of smears showing gonococci when } \\
\cline { 2 - 4 } & \multicolumn{4}{c}{ Endocervical cells } \\
\cline { 2 - 4 } Metaplastic cells & + & - & Total \\
\hline+ & $29(13 / 45)$ & $20(14 / 69)$ & $24(27 / 114)$ \\
Total & $0(0 / 4)$ & $0(0 / 28)$ & $0(0 / 32)$ \\
& $27(13 / 49)$ & $14(14 / 97)$ & $18(27 / 146)$ \\
\hline
\end{tabular}

+ Present - absent

RELIABILITY BETWEEN OBSERVERS

Specificity was high when the findings of the eight observers were compared with the cervical culture results (table IV). The eight cytotechnologists scored the slides from 10 culture-negative patients as positive or negative for the presence of diplococci $(80$ determinations). The highest specificity would have occurred if all the scores had been negative. A total of $78(98 \%)$ negative observations was recorded. The sensitivity, on the other hand, was very low.

The eight observers scored the slides from 10 culture-positive patients as positive or negative. Of a possible 80 positive scores (highest sensitivity) only 35 $(44 \%)$ positive observations were made. Again, the high specificity occurred because the observers identified the results of most cases as negative for diplococci.
TABLE IV Correlation of the presence of diplococci on Papanicolaou smears with the results of cervical culture

\begin{tabular}{|c|c|c|c|}
\hline \multirow[b]{2}{*}{ Patient No } & \multicolumn{2}{|c|}{$\begin{array}{c}\text { No of observers detecting } \\
\text { diplococci on smears* }\end{array}$} & \multirow[b]{2}{*}{ Culture results } \\
\hline & Present & Absent & \\
\hline 1 & 1 & 7 & + \\
\hline 2 & 6 & 2 & + \\
\hline 3 & 1 & 7 & - \\
\hline 4 & 0 & 8 & - \\
\hline 5 & 2 & 6 & + \\
\hline 6 & 3 & 5 & + \\
\hline 7 & 6 & 2 & + \\
\hline 8 & 0 & 8 & - \\
\hline 9 & 2 & 6 & + \\
\hline 10 & 0 & 8 & - \\
\hline 11 & 0 & 8 & - \\
\hline 12 & 0 & 8 & - \\
\hline 13 & 0 & 8 & - \\
\hline 14 & 6 & 2 & + \\
\hline 15 & 2 & 6 & + \\
\hline 16 & 1 & 7 & - \\
\hline 17 & 0 & 8 & - \\
\hline 18 & 3 & 5 & + \\
\hline 19 & 4 & 4 & + \\
\hline 20 & 0 & 8 & - \\
\hline
\end{tabular}

*All eight observers reviewed each slide independently

+ Positive - negative

Although the number of endocervical cells (EC) or metaplastic cells (MC) actually present in the smears from 10 patients with positive gonococcal culture results were not measured, it was possible to assess the agreement among the eight observers for the presence or absence of these cells in the 20 smears. If the cytotechnologists agreed perfectly, a case would be scored as either EC/MC-positive or EC/MCnegative by all eight observers. The number of cases that were scored EC-positive by zero to eight observers is given in table $\mathrm{V}$. Very poor agreement was found among the observers (table V). For nine cases, four observers scored EC-positive whereas the other four scored EC-negative. The presence of metaplastic cells on the slides is also shown in table V. Although observers scored MC-positive more often than they scored EC-positive, agreement was again poor.

\section{Discussion}

Several parameters are important for evaluating a procedure as an effective screening test. Validity, which provides a good preliminary indication of the individuals who either have or do not have the disease, has two components. Sensitivity is the ability to identify correctly those who have the disease and specificity is the ability to identify correctly those who do not have the disease. In this study, sensitivity and specificity were measured by comparing an observer's skill in recognising diplococci on the Papanicolaou smears with the results of cervical 
TABLE V Variability between findings of eight observers of the presence of endocervical and metaplastic cells on Papanicolaou smears of 20 patients $(10$ with positive culture results for $N$ gonorrhoeae, 10 with negative results)

\begin{tabular}{lll}
\hline \multirow{2}{*}{$\begin{array}{l}\text { No of observers } \\
\text { reporting presence } \\
\text { of cells }\end{array}$} & \begin{tabular}{l} 
No of cases reported with either: \\
\cline { 2 - 3 }$(E C+)$
\end{tabular} & $\begin{array}{l}\text { Metaplastic cells } \\
(M C+)\end{array}$ \\
\hline 0 & $4^{*}$ & 0 \\
1 & 2 & 0 \\
2 & 2 & 1 \\
3 & 1 & 4 \\
4 & 9 & 1 \\
5 & 1 & 3 \\
6 & 0 & 4 \\
7 & 0 & 3 \\
8 & 1 & 4 \\
\hline
\end{tabular}

+ Positive

*In each of four cases, no observers reported the presence of endocervical cells (example)

cultures. Cervical culture was accepted as the definitive diagnostic procedure even though it may identify a suspected gonococcal carrier in approximately $90 \%$ of cases. ${ }^{7}$ Our data indicated that the sensitivity of the Papanicolaou smear in identifying diplococci was only $18 \%$. Its validity as a screening test was therefore poor.

Yield-the amount of previously unrecognised disease diagnosed and brought to treatment-is another important parameter for evaluating a screening test. The United States Public Health Service reported that, during 1977, 8456410 women had cultures for gonorrhoea performed in a variety of health care facilities. In this population, 393305 $(4 \cdot 7 \%)$ women had positive culture results for $N$ gonorrhoeae. ${ }^{1}$ Our study could not quantitate yield; however, if the Papanicolaou smear had been used to screen these women for gonorrhoea, a sensitivity of $18 \%$ would have detected only 70795 cases. Yield therefore would have been very low.

A screening technique must give consistent results when it is performed more than once under the same conditions on the same individual to be considered precise or reliable. In this study, variability among the eight observers who reviewed the same 20 slides was high. Thus, interobserver reliability was poor. This may be partly due to the unsuitability of the Papanicolaou stain for identifying gonococci. Since the stain is not a Gram stain, most bacteria stain a similar bluish colour, and it is not possible to distinguish between Gram-positive and Gramnegative organisms. Diplococci are also difficult to recognise because oil immersion lenses, which are necessary to identify them, are not routinely used in cytodiagnosis.

It is interesting to note that reliability between observers showed a low sensitivity even though the cytotechnologists spent an excessive amount of time searching for the diplococci (table I). The time required to screen each case was so great that this method would be impractical for detecting diplococci, even if the reliability between observers had been excellent. Thin et $a l^{8}$ evaluated the Papanicolaou smear as a method for detecting Candida sp. They also concluded that the time necessary for prolonged examination of the smears was excessive. ${ }^{8}$

Previous investigators ${ }^{34}$ have reported that the presence of metaplastic or endocervical cells or both on the smear greatly facilitated identification of the diplococci. Our data did not support this conclusion. Furthermore, our study showed that the finding of endocervical or metaplastic cells on the slides from 10 women with positive cervical culture results varied greatly among the eight cytotechnologists (table V). Although these authors ${ }^{34}$ also reported that more than $50 \%$ of routine Papanicolaou smears were unsuitable for screening owing to inadequate metaplastic or endocervical cells, the eight observers at the New York Hospital did not find this a problem.

The possibility of using an already widely accepted diagnostic technique, such as the cytological smear, as a means of screening for gonococci is attractive. Unfortunately, this study suggests that the excessive time taken to examine the slides, poor interobserver reliability, and a low sensitivity for finding diplococci make the Papanicolaou smear an impractical method for detecting gonorrhoea in asymptomatic women.

We wish to thank Jane Bancroft, $M$ Carroll Pariseau, Barbara Topf, and the cytotechnologists of the Papanicolaou Cytology Laboratory for their assistance.

\section{References}

1. US Department of Health Education and Welfare. STD Fact Sheet, 34th edn. HEW Publication No 79-8195, Atlanta, Georgia: CDC, 1979;2:34.

2. Schmale JD, Martin jun JE, Domescik G. Observations on the culture diagnosis of gonorrhea in women. JAMA 1969;210: 312-4.

3. Arsenault GM, Kalman CF, Sorensen KW, Craig JD. The Papanicolaou smear as a technique for gonorrhea detection: a feasibility study. J Am Vener Dis Assoc 1976;2:35-8.

4. Heller CJ. Neisseria gonorrhoeae in Papanicolaou smears. Acta Cytol 1974; 18:338-40.

5. Lynnette EH, Spaulding EH, Truant JP. Manual of Clinical Microbiology. 2nd edn. Washington DC: American Society of Microbiology, 1974: 124-9.

6. Papanicolaou GN. Atlas of Exfoliative Cytology. Cambridge Mass: Harvard University Press, 1954:8.

7. Caldwell JG, Price EV, Pazin GJ, Cornelius III CE: Sensitivity and reproducibility of Thayer-Martin culture medium in diagnosing gonorrhea in women. Am J Obstet Gynecol 1971; 109:463-8

8. Thin RNT, Atia W, Parker JDJ, Nicol CS, Canti G. Value of Papanicolaou-stained smears in the diagnosis of trichomoniasis, candidiasis, and cervical herpes simplex virus infection in women. Br J Vener Dis 1975;51:116-8. 